\title{
Validez diagnóstica del GeneXpert para Mycobacterium tuberculosis y prueba de resistencia a rifampicina
}

\author{
DOI : $10.5377 /$ alerta.v4i3.8829 \\ Ana Carolina Lacayo de Santana ${ }^{1^{*}}$, Polyta Gabriela Rodríguez $\mathrm{Cruz}^{2}$, Zayda Olivia Pérez Aguilar ${ }^{3}$, \\ Cecilia Vásquez Cornejo ${ }^{4}$ \\ 1. Hospital Policlínico Zacamil del Instituto Salvadoreño del Seguro Social. \\ 2. Hospital Nacional de la Mujer Dra. María Isabel Rodríguez. \\ 3. Unidad Comunitaria de Salud Familiar Intermedia El Paisnal, Región Metropolitana, Ministerio de Salud. \\ 4. Hospital Nacional de Niños Benjamín Bloom, Ministerio de Salud. \\ ${ }^{*}$ Correspondencia \\ $\square$ carolacayo@hotmail.com
}

1. (1) 0000-0001-8893-5780

2. (1) 0000-0002-2069-9176

3. (1) 0000-0002-9397-1046

4. (1) 0000-0002-9061-9266

\section{G} ACCESO ABIERTO

Diagnostic validity of the GeneXpert for Mycobacterium tuberculosis and rifampicin resistance test

\section{Citación recomendada:}

Lacayo de Santana AC,

Rodríguez Cruz PG, Pérez

Aguilar ZO, Vásquez

Cornejo C. Validez

diagnóstica del GeneXpert para Mycobacterium

Tuberculosis y prueba de resistencia a rifampicina.

Alerta.2021:4(3):176-180

DOI: 10.5377/alerta.v4i3.8829

Recibido:

02 de Octubre de 2020

\section{Aceptado:}

20 de febrero de 2021

Publicado:

26 de julio de 2021

\section{Contribución de autoría:} ACLDS': diseño de la investigación, búsqueda bibliográfica, análisis de los datos y redacción del manuscrito. PGRC²: análisis de los datos y redacción del manuscrito. ZOPA ${ }^{3}$ : análisis de los datos y redacción del manuscrito. CVC ${ }^{4}$ : búsqueda bibliográfica, análisis de los datos y redacción de manuscrito.

\section{Conflicto de intereses:}

Los autores declaran no tener conflictos de interés en el estudio.

\section{Resumen}

Introducción. El diagnóstico temprano de tuberculosis permite el control de la enfermedad y su transmisibilidad. Objetivo. Describir la validez diagnóstica del GeneXpert MTB/RIF para Mycobacterium tuberculosis en muestra bronquial, utilizando como referencia el cultivo Löwenstein Jensen. Metodología. Estudio transversal analítico, mediante revisión de 942 registros de la Unidad de Broncoscopía durante el año 2014 al 2018, de las cuales 320 cumplieron criterios de inclusión. Estos datos fueron exportados a un formato compatible con Epi Info versión 7 y analizados con parámetros estadísticos de sensibilidad, especificidad, valor predictivo positivo, valor predictivo negativo, prueba de concordancia e índice Kappa Epidat 4,2. Resultados. De los 320 pacientes sometidos a fibrobroncoscopía diagnóstica para tuberculosis con GeneXpert MTB/RIF, los resultados negativos fueron 79\% (252) y positivo $21 \%$ (68); el $1 \%$ mostró resistencia a rifampicina. Se reportó una fuerte concordancia de GeneXpert MTB/RIF con el cultivo bacilo ácido alcohol resistente, que se determinó con un índice de kappa de 0,88 +/- $(0,81-0,94)$ IC 95\%, una sensibilidad del $98 \%$, especificidad del $96 \%$, valor predictivo positivo $83 \%$ (IC 95\%), valor predictivo negativo 99,6\% (CI 95\%). Conclusión. La prueba GeneXpert MTB/RIF tiene una capacidad altamente sensible y específica para el diagnóstico de tuberculosis en muestras obtenidas por fibrobroncoscopía.

Palabras clave

Tuberculosis pulmonar, broncoscopía, técnicas de diagnósticomolecular

\section{Abstract}

Introduction. The early diagnosis of tuberculosis allows the control of the disease and its transmissibility. Objective. Describe the diagnostic validity of GeneXpert MTB / RIF for Mycobacterium tuberculosis in bronchial sample using the Löwenstein Jensen culture as reference. Methodology. Analytical cross-sectional study, through a review of 942 records of the Bronchoscopy Unit during the year 2014 to 2018, of which 320 met inclusion criteria. These data were exported to a format compatible with Epi Info version 7, analyzed with statistical parameters of sensitivity, specificity, positive predictive value, negative predictive value, concordance test, and Kappa Epidat index 4,2. Results. Of the 320 patients who underwent diagnostic bronchoscopy for tuberculosis with GeneXpert MTB / RIF, the negative results were 79\% (252), and positive $21 \%(68), 1 \%$ showed genetic resistance to rifampicin. A strong concordance of GeneXpert MTB / RIF was reported with the acid-alcohol-resistant bacillus culture determined with a kappa index of 0,88 +/- $(0,81-0,94) 95 \% \mathrm{Cl}$, a sensitivity of $98 \%$, specificity $96 \%$, positive predictive value $83 \%$ (95\% Cl), negative predictive value $99,6 \%$ (95\% Cl). Conclusions. The GeneXpert MTB / RIF Test has a highly sensitive and specific capacity for the diagnosis of tuberculosis in samples obtained by bronchoscopy.

Keywords

Pulmonary tuberculosis, bronchoscopy, molecular diagnostic techniques 


\section{Introducción}

La tuberculosis es un problema de salud pública sobre todo en países con bajo nivel de desarrollo humano'. Afecta a poblaciones vulnerables, entre ellos, privados de libertad, indigentes y otros sectores excluidos ${ }^{2}$. El diagnóstico temprano de tuberculosis activa sigue siendo un reto para los sistemas de salud, a pesar de contar con diferentes estrategias para su detección $n^{3}$.

El control sobre la cadena de transmisión se ve afectado no solo por el retraso en el diagnóstico temprano, sino también por el inicio tardío del tratamiento, factores de proximidad, prevalencia de la enfermedad, factores ambientales y factores intrínsecos de la persona expuesta. Estos aspectos impiden el adecuado funcionamiento de las defensas como huésped contra la infección y la enfermedad ${ }^{3}$.

En el 2018, la Organización Panamericana de la Salud (OPS) calculó que un error del $0,5 \%$ en el diagnóstico implica un incremento de 1327097 USD, en el gasto del Programa Nacional de Tuberculosis y la aparición de 183 nuevos casos $^{4}$.

La prueba semicuantitativa GeneXpert MTB/RIF de diagnóstico in vitro que detecta componentes del genoma del Mycobacterium tuberculosis (MTB) y resistencia a rifampicina (RIF) se realiza de forma automatizada, purificando y amplificando ácidos nucleicos, identificando la secuencia diana a través de ensayos de reacción en cadena de la polimerasa (PCR) y de PCR con transcriptasa inversa (RT-PCR), ambas en tiempo real. Esta prueba contiene cebadores que amplifican una porción del gen rpoB y sondas que distinguen la secuencia mutada asociada a la resistencia a rifampicina con resultados en dos horas ${ }^{4-6}$.

Se necesita de un instrumento que contiene un ordenador personal y software precargado para realizar las pruebas y ver los resultados (MTB detectado o no detectado, RIF resistencia detectada, indeterminada o no detectada), así como un cartucho autónomo desechable de un solo uso que contienen los reactivos RT-PCR y PCR ${ }^{4-6}$.

En los últimos años, esta prueba se ha convertido en una opción que no requiere mayor entrenamiento para el personal, con resultados en tiempo, de utilidad en pacientes que presentan una baja carga bacilar en las muestras para lectura de frotis bacilo ácido alcohol resistente (baciloscopía) con Zielh Neelsen, que pueden reportarse negativas y posterior a 60 días positivas en el cultivo Löwenstein Jensen. Aunque la baciloscopía representa una prueba de menor costo que también brinda resultados inmediatos requiere de personal altamente ca- pacitado para su lectura, lo que finalmente demanda inversión ${ }^{10-12}$.

Fabricantes de la prueba GeneXpert MTB/ RIF describen su rendimiento con sensibilidad del $76,1 \%$ y una especificidad del $98,8 \%$ frente al cultivo Löwenstein Jensen con baciloscopías negativas 5 . El presente estudio describe la validez diagnóstica que la prueba GeneXpert MTB/RIF provee en el diagnóstico de tuberculosis pulmonar utilizando muestras obtenidas de forma directa por broncoscopía ante la dificultad del diagnóstico convencional por baciloscopía y utilizando como referencia el método de Petroff modificado en cultivo Löwenstein Jensen.

\section{Metodología}

El estudio se llevó a cabo con método transversal analítico retrospectivo que consistió en el análisis secundario de los registros de pacientes sometidos a fibrobroncospía diagnóstica en la Unidad de Broncoscopía del Hospital Nacional de Neumología y Medicina Familiar Dr. José Antonio Saldaña, ubicado en el municipio de Panchimalco del departamento de San Salvador. El período de ejecución de la investigación fue de enero de 2014 a diciembre de 2018.

La población estuvo conformada por 942 pacientes. Solamente se seleccionaron a quienes se les realizó tanto a prueba de GeneXpert MTB/RIF como cultivo Löwenstein Jensen simultáneamente, quedando un total de 320 pacientes.

La información recolectada fue digitada en una hoja de cálculo de Microsoft Office Excel. Posteriormente, la base de datos fue exportada a un formato compatible con Epi Info versión 7 para el cálculo de las frecuencias de las variables edad, sexo, ocupación, hallazgos radiológicos e indicaciones de fibrobroncoscopía diagnóstica. Se calcularon valores absolutos y proporciones.

Finalmente, se realizó el análisis de los resultados obtenidos con el GeneXpert MTB/ RIF y los resultados del cultivo Löwenstein Jensen. Los datos fueron analizados con parámetros estadísticos de sensibilidad, especificidad, valor predictivo positivo, valor predictivo negativo, probabilidad/Odds pretest, probabilidad/Odds post-test, coeficiente de verosimilitud positivo, coeficiente de verosimilitud negativo, prueba de concordancia e índice Kappa. Para el análisis de los datos se utilizó Epidat 4,2 Xunta de Galicia.

Previo a la ejecución de la investigación se obtuvo la aprobación por parte del Comité de Ética del Hospital Nacional de Neumología y Medicina Familiar Dr. José Antonio Saldaña. La base de datos fue de uso exclusivo del equipo de investigación. 


\section{Resultados}

Pacientes sometidos al procedimiento diagnóstico es $51 \%$ del sexo masculino $y$, sin embargo, al establecer el diagnóstico bacteriológico se observa que $52 \%$ representa el sexo femenino. La edad media del total de pacientes que se sometieron a la fibrobroncoscopía diagnóstica fue de 47,7+/-19,3 IC $95 \%$ (RI 29.33) y la de los pacientes catalogados como tuberculosis pulmonar por bacteriología fue de 43,9+/-18,7 IC $95 \%$.

En cuanto a la ocupación de los pacientes a los que se les realizó el estudio, se observó que (96) 30\% de los pacientes pertenecen al rubro de ama de casa, seguida de trabajador del campo (31) 10\%, estudiante (28) 9\% y comerciante (23) 7\%. Al relacionarla con el diagnóstico bacteriológico la mayoría continúa siendo ama de casa con (19) $28 \%$, seguida por igual porcentaje para trabajador del campo (8) $12 \%$ y comerciante (8) $12 \%$.

De los pacientes que presentaban comorbilidades crónicas, el 58\% reportó ser $\mathrm{VIH}$ positivo y el $23 \%$ tener diagnóstico de diabetes mellitus previo al procedimiento. Al relacionarse con el diagnóstico bacteriológico, el $9 \%$ registra padecer diabetes mellitus y el $3 \% \mathrm{VIH}$.

La sospecha clínica de tuberculosis con $62 \%$ representó la indicación de fibrobroncoscopía diagnostica más frecuente, seguida de los hallazgos radiológicos con el $26 \%$.

La sospecha clínica de tuberculosis con $62 \%$ representó la indicación de fibrobroncoscopía diagnostica más frecuente, seguida de los hallazgos radiológicos con el $26 \%$.

El $21 \%$ de los pacientes reporta detección del Mycobacterium tuberculosis por medio de la prueba GeneXpert MTB/RIF y el $18 \%$ reportó crecimiento del bacilo en el cultivo Löwenstein Jensen.

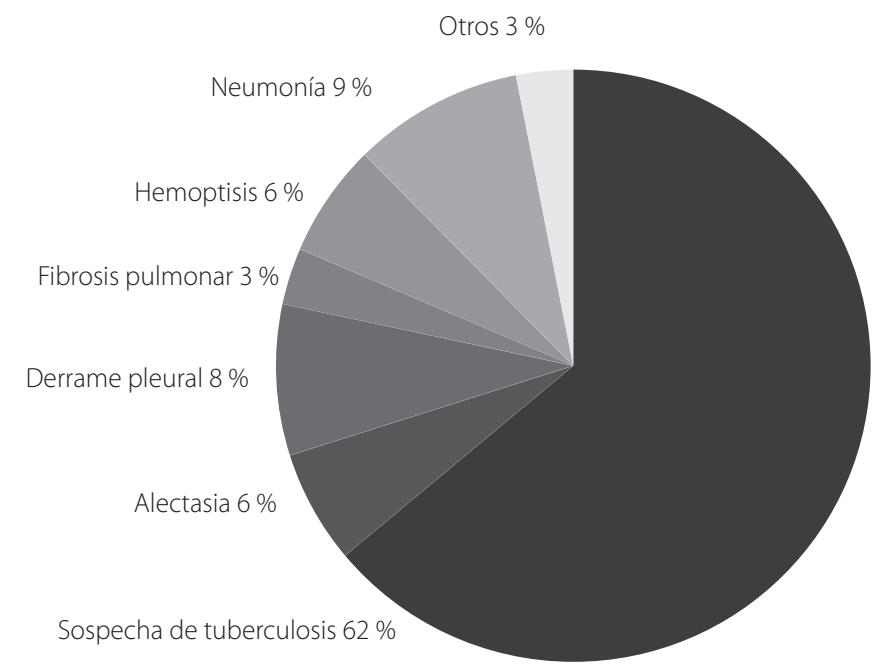

Figura 1. Indicación de fibrobroncoscopía en el Hospital Nacional de Neumología y Medicina Familiar Dr. José Antonio Saldaña 2014 a 2018

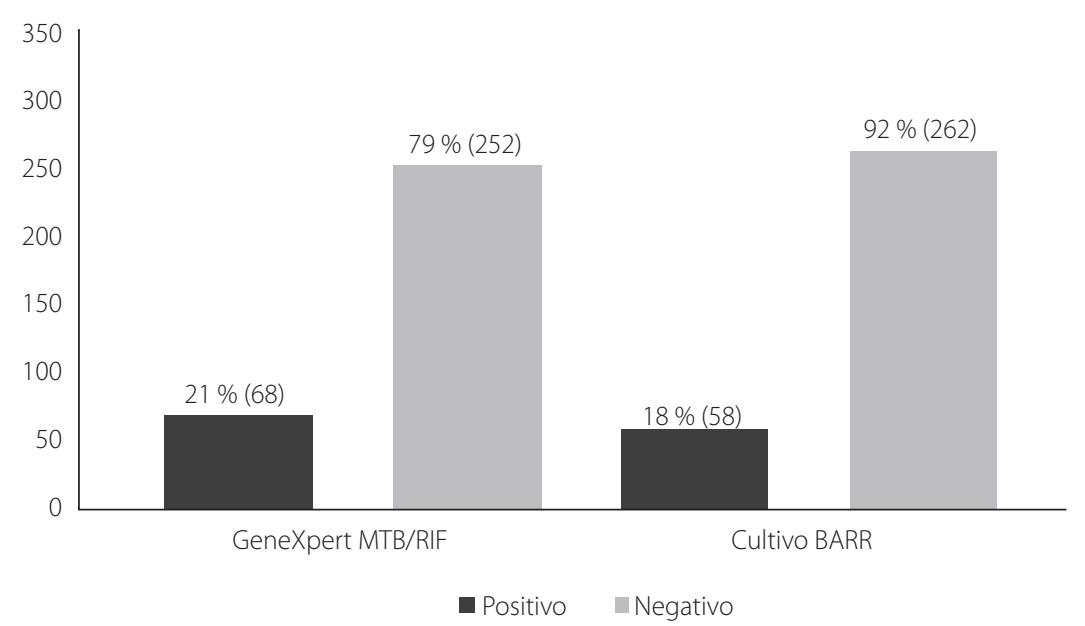

Figura 2. Resultados de GeneXpert MTB/RIF y Cultivo BARR de muestras extraídas a los pacientes por fibrobroncoscopía diagnóstica en el Hospital Nacional de Neumología y Medicina Familiar Dr. José Antonio Saldaña, 2014 a 2018 
Tabla 1. Concordancia e Índice Kappa de Cohen

\begin{tabular}{lc}
\hline Ítem & Valor \\
\hline Kappa & 0,8816 \\
EE* $^{*}$ & 0,0333 \\
IC $(95,0 \%)$ & $0,8163-0,9469$ \\
Estadístico Z & 15.85 \\
Valor P & 0,0000 \\
Sensibilidad & $98 \%$ \\
Especificidad & $96 \%$ \\
VPP & $83 \%$ \\
VPN & $99,6 \%$ \\
Nivel de confianza & $95 \%$ \\
\hline
\end{tabular}

EE: error estándar

VPP: valor predictivo positivo

VPN: valor predictivo negativo

\section{Discusión}

Se identifica que existe validez diagnóstica del GeneXpert MTB/RIF en detecciones del Mycobacterium tuberculosis (MTB) en muestras tomadas de forma directa del árbol bronquial por fibrobroncoscopía, siendo similar a los confirmados por cultivo BARR. En el cálculo de la concordancia de resultados de la prueba GeneXpert MTB/RIF frente a cultivo Löwenstein Jensen respecto al azar con el Índice Kappa de Cohen $(K)=0,88$, mostró una concordancia clasificada como muy buena (casi perfecto), similar al estudio en Chile $(K)=0,96^{13}$.

La prueba de GeneXpert tiene una sensibilidad de $98 \%$ (IC 95\%), similar a las observadas en Pakistán (78/85) $(92 \%)^{14}$, India $(155 / 170)(91 \%)^{6}$, Corea del Sur (35/43) $(81,6 \%)^{15}$, Chile $(31 / 33)(94 \%)^{16}$ y Sudáfrica $(25 / 27)(93 \%)^{17}$ y una especificidad del $96 \%$ (IC $95 \%)$, muy parecida a la encontrada por Lee $(97 / 97)(100 \%)^{15}$, Vallejo (334/351) $(95 \%)^{16}$ y Theron $(127 / 133)(96 \%)^{17}$.

Se reportaron 10 resultados de falsos positivos y 1 resultado de falso negativo por GeneXpert MTB/RIF. Las repercusiones más frecuentes al diagnosticarse tuberculosis sin padecerla son las psicosociales y los efectos adversos de los fármacos utilizados en el tratamiento. En cambio, el impacto de no detectar la enfermedad es mayor debido a las complicaciones del curso natural de la enfermedad y su transmisibilidad.

Otros indicadores del rendimiento de GeneXpert MTB/RIF como el valor predictivo positivo (VPP) $83 \%$ (IC 95\%) y valor predictivo negativo (VPN) 99,6\% (IC 95\%) son acordes a los reportes de Theron (VPP $83 \%$ VPN $98 \%)^{17}$ y Bernard (VPP $80 \%$ VPN $95,5 \%)^{18}$.

Los pacientes con la comorbilidad VIH se sometieron en mayor proporción al exa- men; sin embargo, aquellos que padecen diabetes mellitus fueron los más asociados al diagnóstico bacteriológico ${ }^{19}$.

La causa más frecuente para indicación de la fibrobroncoscopía diagnóstica fue la sospecha clínica de tuberculosis pulmonar, la cual no se pudo establecer con los métodos diagnósticos convencionales y los hallazgos de la radiografía de tórax ${ }^{20}$.

Los pacientes que se sometieron al procedimiento y que se les estableció o se descartó el diagnóstico de tuberculosis no presentan diferencia significativa en cuanto al sexo, edad y ocupación. La población estudiada presenta una edad dispersa, tal como lo muestran estudios realizado en $\mathrm{Pa}$ kistán y China ${ }^{9},{ }^{10}$. Esto puede deberse a que la TB es una enfermedad en la que participan diferentes factores de riesgo, entre ellos demográficos y socioeconómicos, los cuales no tienen un papel individual establecido.

Una de las limitantes de esta investigación fueron los insuficientes datos sobre las características y comportamiento de la población que puede conferir factores de riesgos y antecedentes médicos relacionados con la enfermedad.

En futuras investigaciones es importante registrar el desempeño diagnóstico del GeneXpert MTB/RIF en esputo espontáneo y las variantes relacionadas a la calidad de la muestra.

\section{Conclusiones}

La prueba molecular GeneXpert MTB/RIF es válida para el diagnóstico de tuberculosis pulmonar en muestras obtenidas por fibrobroncoscopía, siendo confiable su uso para establecer diagnóstico temprano por su sensibilidad y especificidad.

En la población estudiada no se encontró una resistencia significativa a la rifampicina, ya que no se reportó en el estudio de sensibilidad del cultivo. La principal comorbilidad asociada es la diabetes mellitus. La tuberculosis se comporta de manera amplia y diversa en la población, ya que se presenta en personas de ambos sexos de todas las edades.

\section{Referencias bibliográficas}

1. Okhovat-Isfahani B, Bitaraf S, Ali Mansournia $M$, Doosti-Irani A. Inequality in the global incidence and prevalence of tuberculosis (TB) and TB/HIV according to the human development index. Medical Journal of the Islamic Republic of Iran. 2019;33:45. DOI: 10.34171/mjiri.33.45 
2. Ayala G, Garay J, Aragon M, Decroo T, Zachariah R. Trends in tuberculosis notification and treatment outcomes in prisons: a country-wide assessment in El Salvador from 2009-2014. Revista Panamericana de Salud Pública. Enero 2016; 39(1):38-43. Disponible en: https://pubmed. ncbi.nlm.nih.gov/27754539/

3. Ministerio de Salud. Norma técnica para la prevención y control de la tuberculosis. San Salvador. 2020. 29 p. Disponible en: https:// bit.ly/357dfaa

4. Organismo Andino de Salud - Convenio Hipólito Unanue. Costo efectividad en el diagnóstico de la tuberculosis. Lima. 76 p. Disponible en: https://bit.ly/2SnzDcB

5. Cepheid ${ }^{\oplus}$ Xpert MTB RIF Assay August 2019 https://www.cepheid.com/Package\%20 Insert\%20Files/Xpert-MTB-RIF-ENGLISHPackage-Insert-301-1404-Rev-F.pdf

6. Patil S, Narwade S, Mirza M. Bronchial Wash Gene Xpert MTB/RIF in Lower Lung Field Tuberculosis: Sensitive, Superior, and Rapid in Comparison with Conventional Diagnostic Techniques. J Transl Int Med. 2017;5(3):174-181. DOl: 10.1515/jtim-20170030

7. Şimşek A, Yapııı İ, Babalık M, Şimşek Z, Kolsuz M. Bronchoscopic diagnostic procedures and microbiological examinations in proving endobronchial tuberculosis. J Bras Pneumol. 2016;42(3):191-195. DOl: 10.1590/ S1806-37562015000000134

8. Reyes Núñez N, Luque Crespo E, Santos Morano J, Alfageme Michavila I. Manual de diagnóstico y terapéutica en neumología. 3a edición. Sevilla. Neumosur. Capítulo 10, Broncoscopia diagnóstica. Requisitos, sedación y técnicas. 125-135. Disponible en: https://bit.ly/3cuSbhQ

9. Zuberi FF, Hussain S, Hameed S, Zuberi BF. Role of Bronchial Washing Gene Xpert in Sputum-Scarce Cases of Suspected Pulmonary Tuberculosis. Pak J Med Sci. 2019;35(1):211-214. DOI: 10.12669/ pjms.35.1.107

10. Miller RJ, Casal RF, Lazarus DR, Ost DE, Eapen GA. Flexible Bronchoscopy. Clin Chest Med. 2018;39(1):1-16. DOl: 10.1016/j. ccm.2017.09.002

11. Treatment Action Group. Una guía para activistas sobre Herramientas de Diagnóstico de la Tuberculosis. New York. Treatment Action Group. 34 p. 2017. Disponible en: https://bit.ly/2TbCKo1

12. Valentini I, Lazzari Agli L, Michieletto $L$, Innocenti M, Savoia F, Del Prato B, et al. Competence in flexible bronchoscopy and basic biopsy technique. Panminerva Med. 2019;61 (3):232-248. DOI: 10.23736/S00310808.18.03563-2
13. García P, Balcells ME, Castillo C, Miranda C, Geoffroy E, Román JC, et al. Evaluación de la técnica Xpert ${ }^{\oplus}$ MTB/RIF para la detección de Mycobacterium tuberculosis complex en muestras extra-pulmonares. Rev Chilena Infectol. 2017;34(4):333-339. DOI: 10.4067/ s0716-10182017000400333

14. Khalil KF, Butt T. Diagnostic yield of Bronchoalveolar Lavage gene Xpert in smear-negative and sputum-scarce pulmonary tuberculosis. J Coll Physicians Surg Pak. 2015;25(2):115-8. DOI: 02.2015/ JCPSP.115118

15. Lee HY, Seong MW, Park SS, Hwang S-S, Lee J, Park YS, et al. Diagnostic accuracy of Xpert ${ }^{\oplus}$ MTB/RIF on bronchoscopy specimens in patients with suspected pulmonary tuberculosis. Int J Tuberc Lung Dis. 2013;17(7):917-921. DOI: 10.5588/ ijtld.12.0885

16. Vallejo VP, Rodríguez DJC, Searle MA, Farga CV. Ensayo Xpert MTB/RIF en el diagnóstico de tuberculosis. Rev. chil. enferm. respir. 2015;31(2):127-131. DOI: 10.4067/S071773482015000200010.

17. Theron G, Peter J, Meldau R, Khalfey H, Gina $\mathrm{P}$, Matinyena B, et al. Accuracy and impact of Xpert MTB/RIF for the diagnosis of smearnegative or sputum-scarce tuberculosis using bronchoalveolar lavage fluid. Thorax. 2013;68(11):1043-1051. DOI: 10.1136/ thoraxjnl-2013-203485

18. Barnard DA, Irusen EM, Bruwer JW, Plekker D, Whitelaw AC, Deetlefs JD, et al. The utility of Xpert MTB/RIF performed on bronchial washings obtained in patients with suspected pulmonary tuberculosis in a high prevalence setting. BMC Pulm Med. 2015;15:103. DOI: 10.1186/s12890-0150086-Z

19. Magaña Revelo MA, Rivas Hernández IA, Morales Cruz JC, Alfaro Vásquez MA. Asociación entre tuberculosis y diabetes mellitus en el primer nivel de atención. Alerta 2020; 3(1):13-17 DOl: 10.5377/alerta. v3i1.8741

20. Malacarne J, Heirich AS, Cunha EAT, Kolte IV, Souza-Santos R, Basta PC. Performance of diagnostic tests for pulmonary tuberculosis in indigenous populations in Brazil: the contribution of Rapid Molecular Testing. J Bras Pneumol. 2019; 45(2):e20180185. DOI: 10.1590/1806-3713/e20180185 